\title{
Supporting Information: Spectrally Resolved Surface-Enhanced Raman Scattering Imaging Reveals Plasmon-Mediated Chemical Transformations
}

\author{
Authors \\ Carlos Diego L. de Albuquerque, Chelsea M. Zoltowski, Brian T. Scarpitti, Deben N. Shoup, and Zachary \\ D. Schultz* \\ Department of Chemistry and Biochemistry, The Ohio State University, Columbus, OH, 43210, USA \\ *corresponding author email: Schultz.133@osu.edu
}

Contents:

Methods

Figure S1

Figure S2

S-4

Figure S3

Figure S4.

Figure S5

Figure S6

S-6

Optimized Structure Coordinates. S-7

References S-9 


\section{Methods}

\section{Nanoparticle Synthesis and Characterization}

Gold nanoparticles were synthesized by a seed mediated growth as described previously. ${ }^{1}$ The nanoparticles used for these experiments has an LSPR peak max at 641 $\mathrm{nm}$, as determined by the extinction spectrum (Figure S2). Transmission electron microscopy was used to examine the size and shape of individual nanoparticles (Figure 1B). After characterization, the nanoparticles were functionalized with 4mercaptobenzoic acid (MBA) overnight by addition of 200 micromolar MBA to 5-fold diluted nanoparticles. The ensemble SERS spectrum of nanoparticles in solution after the functionalization with MBA was acquired for 30 s using a Snowy Raman spectrometer with about $23 \mathrm{~mW} 638 \mathrm{~nm}$ laser excitation (Figure S5).

\section{Density-Functional Calculations:}

All calculations were performed through the Ohio Supercomputer Center ${ }^{2}$ with Gaussian 16. ${ }^{3}$ The chemical structure mercaptobenzoic acid (MBA) was built using Gaussview 6. ${ }^{4}$ Optimizations followed by a frequency analyses, including Raman vibrations, were performed on MBA using the CAMB3LYP ${ }^{5}$ functional with a $6-31+\mathrm{G}(\mathrm{d})$ basis $\operatorname{set}^{6-8}$. Optimized stationary points were confirmed to be local minima (all real vibrational frequencies) using vibrational frequency analyses. Solvent effects were simulated in water with the solvation model density (SMD) method with electrostatics in terms of the integral-equation formalism polarizable continuum model (IEF-PCM). ${ }^{9-11}$ The calculated frequencies were scaled by 0.94 to match the aromatic ring mode observed experimentally at $1580 \mathrm{~cm}^{-1}$.

\section{Spectral SERS imaging}

The spectrally resolved SERS imaging microscope was constructed around an Olympus IX-41 inverted microscope. $659 \mathrm{~nm}$ laser excitation was achieved using a 300 $\mathrm{mW}$ single longitudinal mode diode laser (Laser Quantum, San Jose, CA). The output power was attenuated and spread across a $30 \mu \mathrm{m}$ field of view such that the power density onto the sample was $1.5 \mathrm{~kW} \mathrm{~cm}^{-2}$. The laser was directed onto the sample through an $20 \mathrm{x}$ (NA 0.3) objective that could be adjusted in height to control the spot size on the sample. The image was collected using a 100x (NA 1.3) oil immersion objective. The signal was passed out the external camera point and through a $660 \mathrm{~nm}$ long pass filter (Semrock), then a 300 groove/mm visible transmission diffraction grating blazed at $17.5^{\circ}$ (Thorlabs), 
and onto the 2-dimensional CMOS sensor (ORCA-Flash 4.0 V3, Hamamatsu, LTD.). Images were collected using Nikon Elements software.

After functionalization with MBA and characterization, the sample was brought to our homebuilt microscope and the spectrally-resolved SERS images were acquired (Figure 1A). To recover the purest SERS profiles, multivariate curve resolution (MCR) analysis was used to decompose the time evolution SERS spectra. Finally, superresolution SERS fitting was used to co-localize the SIFs within the diffraction-limited spot as described in. ${ }^{12}$

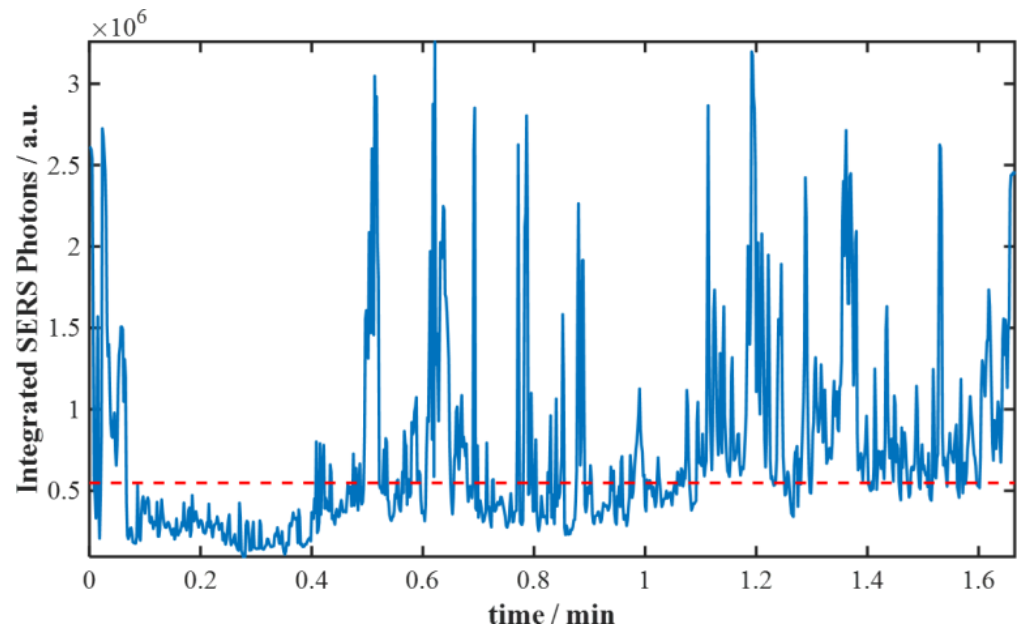

Figure S1. Typical SERS intensity fluctuations (SIFs) calculated by integration of the vibrational modes around $1580 \mathrm{~cm}^{-1}$. The red line indicates the median photon intensity $5.5 \times 10^{5}$ photons s $^{-1}$. 

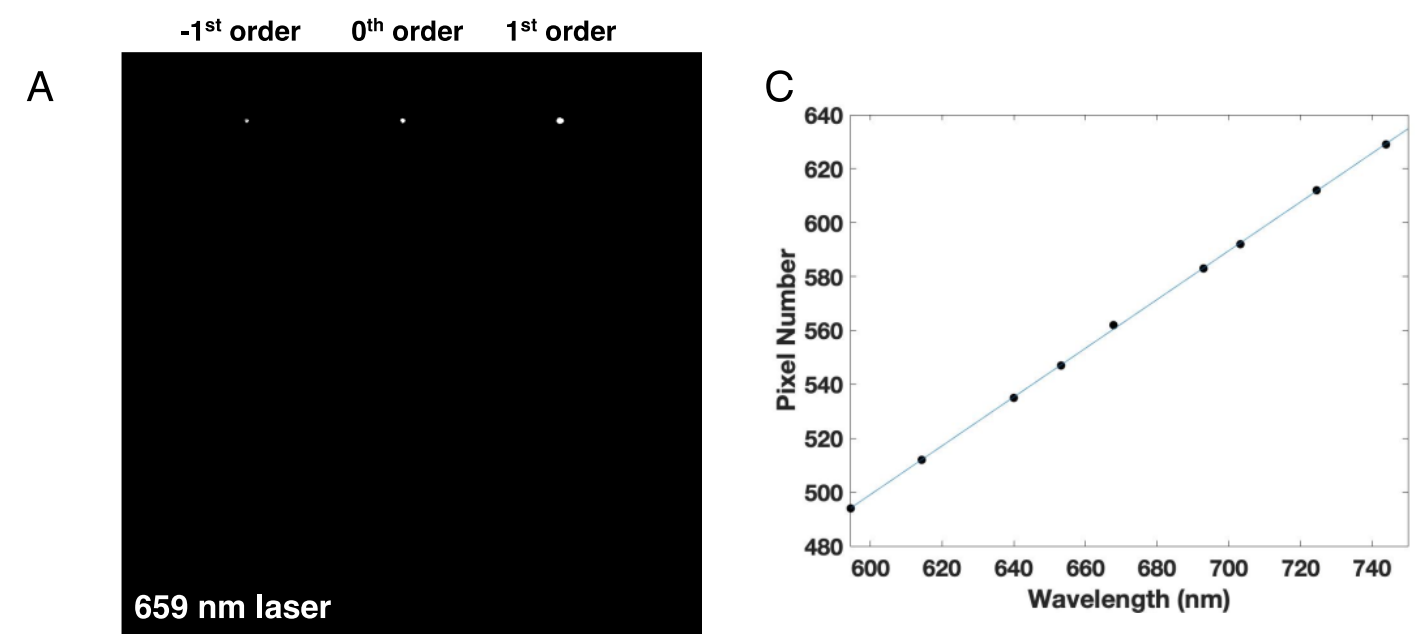

B
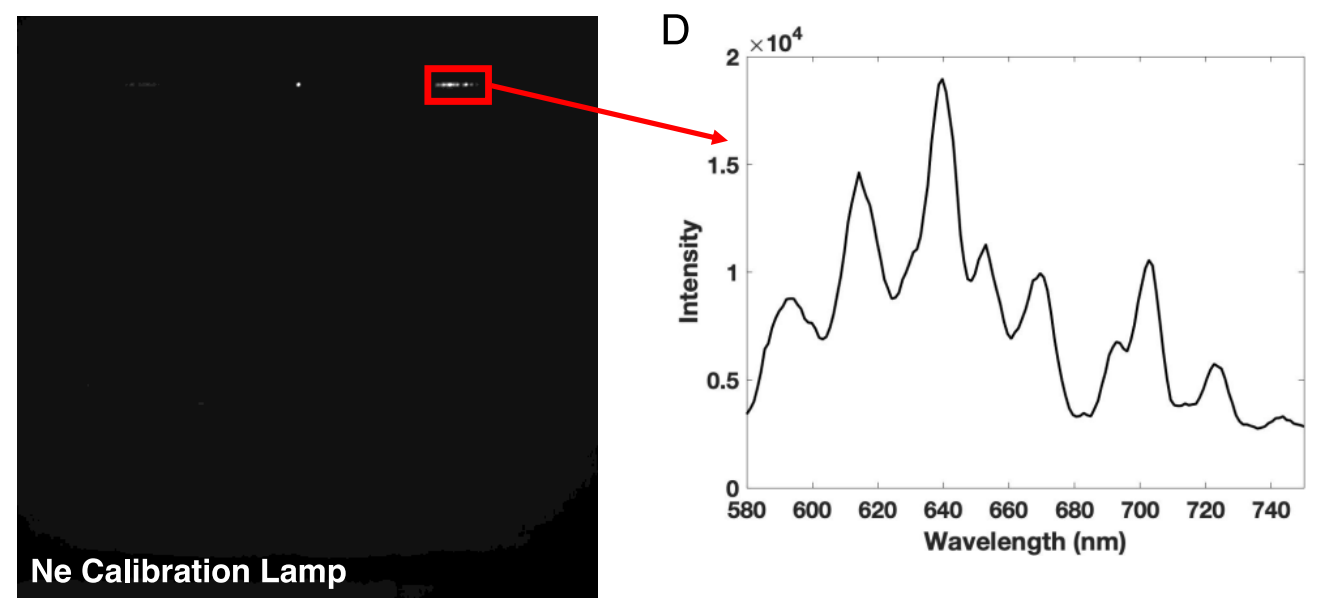

Figure S2. (A) the full frame of the CMOS camera is shown in our spectrally resolved imaging format when the $659 \mathrm{~nm}$ laser is incident on a $5 \mu \mathrm{m}$ pinhole (Thorlabs). (B) a Neon calibration lamp is focused on the distal side of the pinhole and the transmitted light from the $5 \mu \mathrm{m}$ pinhole is imaged on the camera. (C) The peaks observed in the $1^{\text {st }}$ order Ne spectrum are used to determine the correlation between pixel location from the $0^{\text {th }}$ order peak and wavelength. The slope of the calibration line is $1.1 \mathrm{~nm} / \mathrm{pixel}$, which is a good agreement with the calculated spectral resolution of $\sim 1.02 \mathrm{~nm} /$ pixel. (D) the wavelength calibrated spectrum from the Ne calibration image is shown. Of note, the $300 \mathrm{gr} / \mathrm{mm}, 17.5^{\circ}$ groove angle transmission grating is blazed for maximum efficiency near $550 \mathrm{~nm}$ and is positioned $20 \mathrm{~mm}$ from the camera sensor (Figure 1A). 


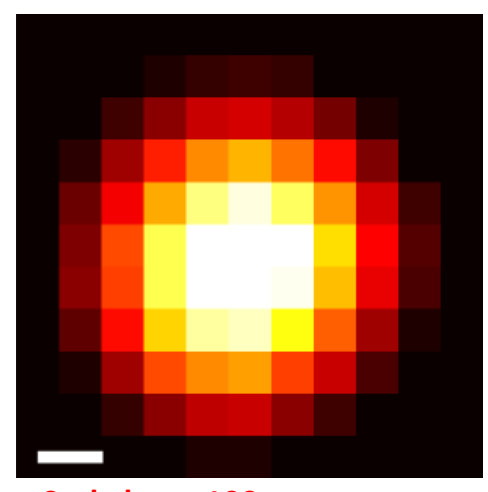

Scale bar $=100 \mathrm{~nm}$

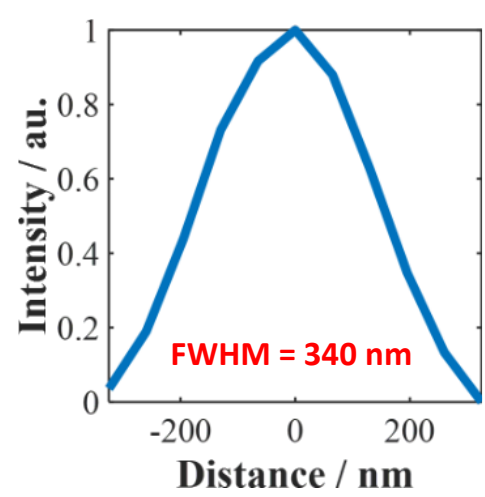

Distance / nm

Figure S3. The image or particle 1 and its corresponding intensity profile across the spot is shown. The Full width at half maximum (FWHM) is $340 \mathrm{~nm}$, which is consistent for a diffraction limited spot with our objective and $659 \mathrm{~nm}$ laser.
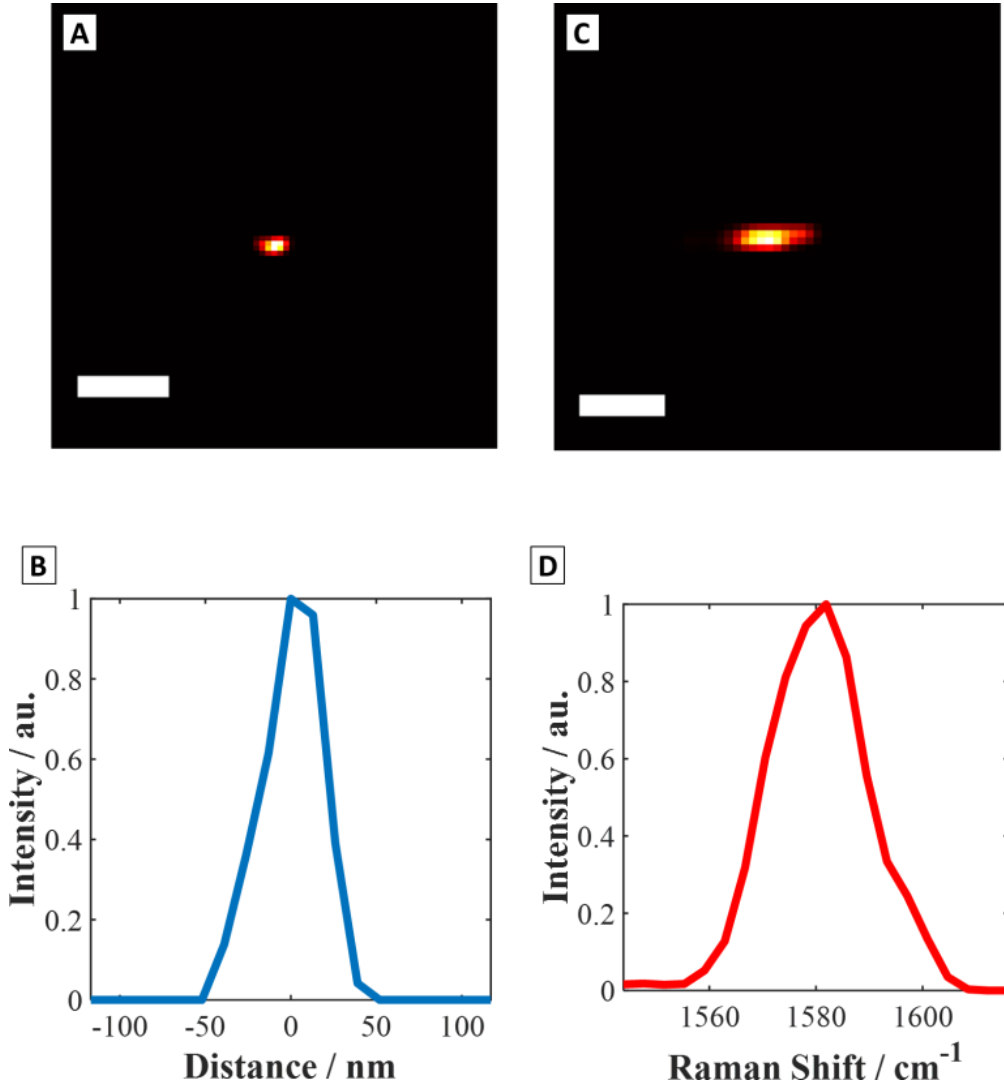

D

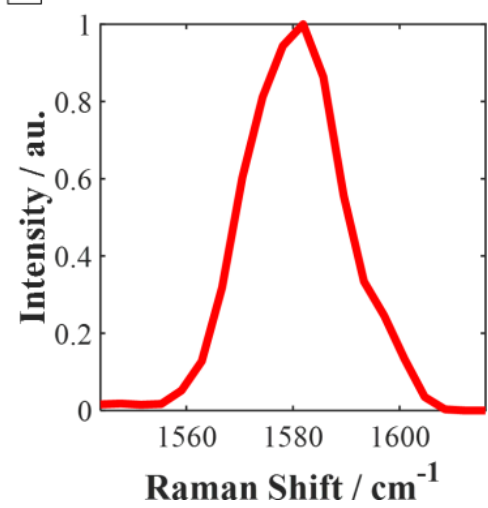

Figure S4. (A) Spatial and (B) Spectral super-resolution SERS mapping obtained from applying localization algorithms on a single nanoparticle imaged in the zeroth order image and from the emission band at $1580 \mathrm{~cm}-1$ in the $n=1$ spectrum. 1000 frames were used to generate the super-resolution results. (C) Spatial and (D) Spectral intensity profile derived the emissions centered at $0 \mathrm{~nm}$ and $1580 \mathrm{~cm}^{-1}$, respectively. The results demonstrated are for nanoparticle highlighted in Figure 1D. The scale bars are $200 \mathrm{~nm}$ for (A) and $40 \mathrm{~cm}^{-1}$ for (C). 


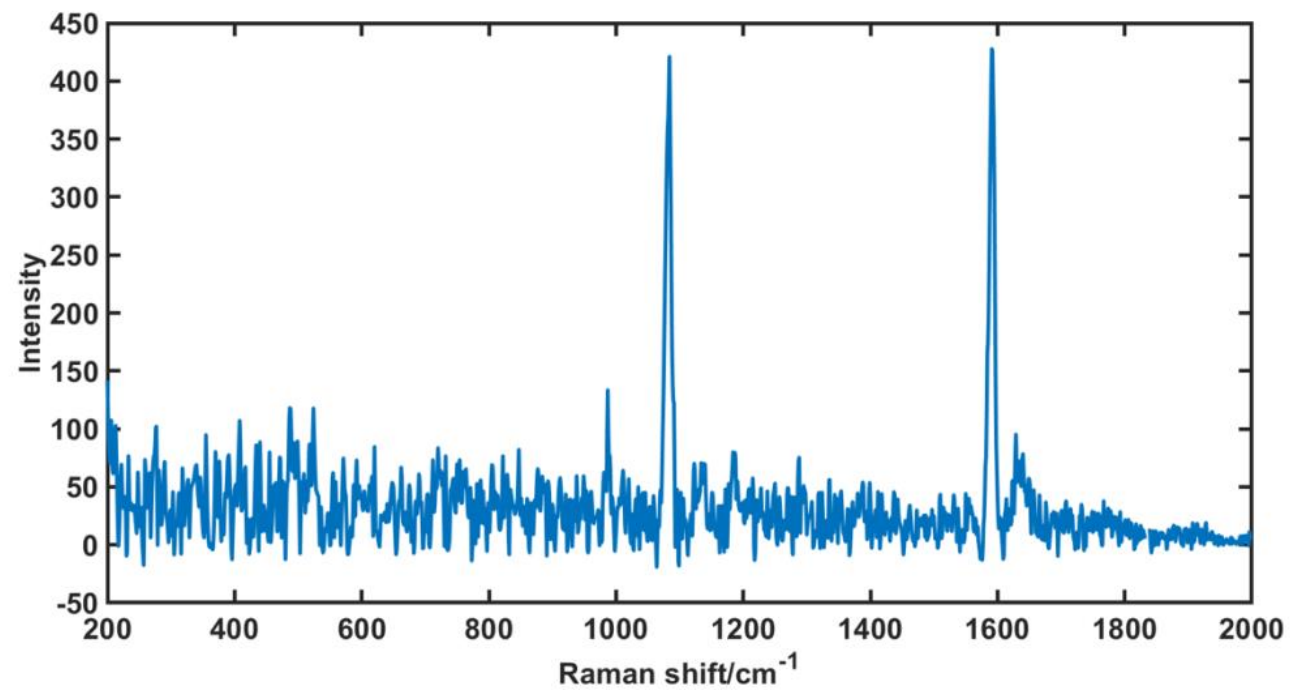

Figure S5. Ensemble SERS spectrum of MBA functionalized nanoparticles used. The spectrum was acquired using $638 \mathrm{~nm}$ laser at $23 \mathrm{~mW}$ for 30 seconds and baseline corrected. The prominent peaks at 1083 and $1580 \mathrm{~cm}^{-1}$ are characteristic of the aromatic ring breathing modes.
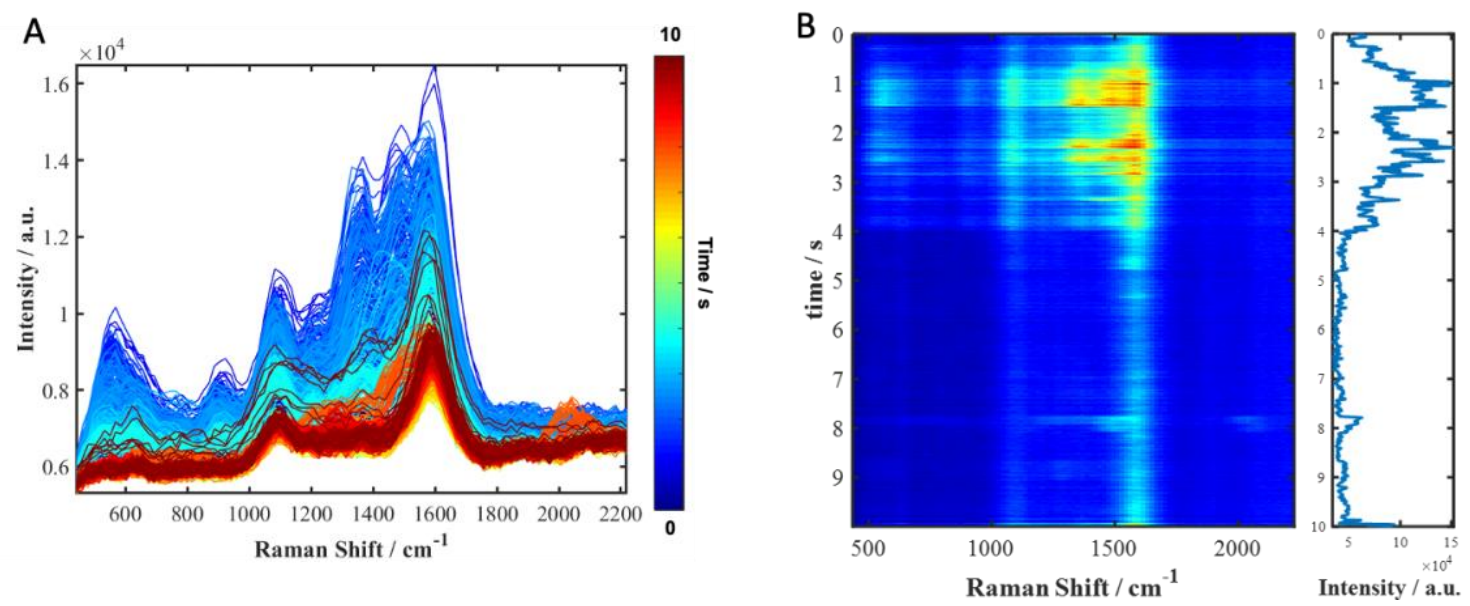

Figure S6. Particle 1 (Figure 1D \& 2) was imaged at frame rate of $100 \mathrm{~Hz}$, shows the same transient signals observed at $10 \mathrm{~Hz}$ and suggests the signal duration in on the order of 10's of milliseconds. 


\section{Optimized structure coordinates:}

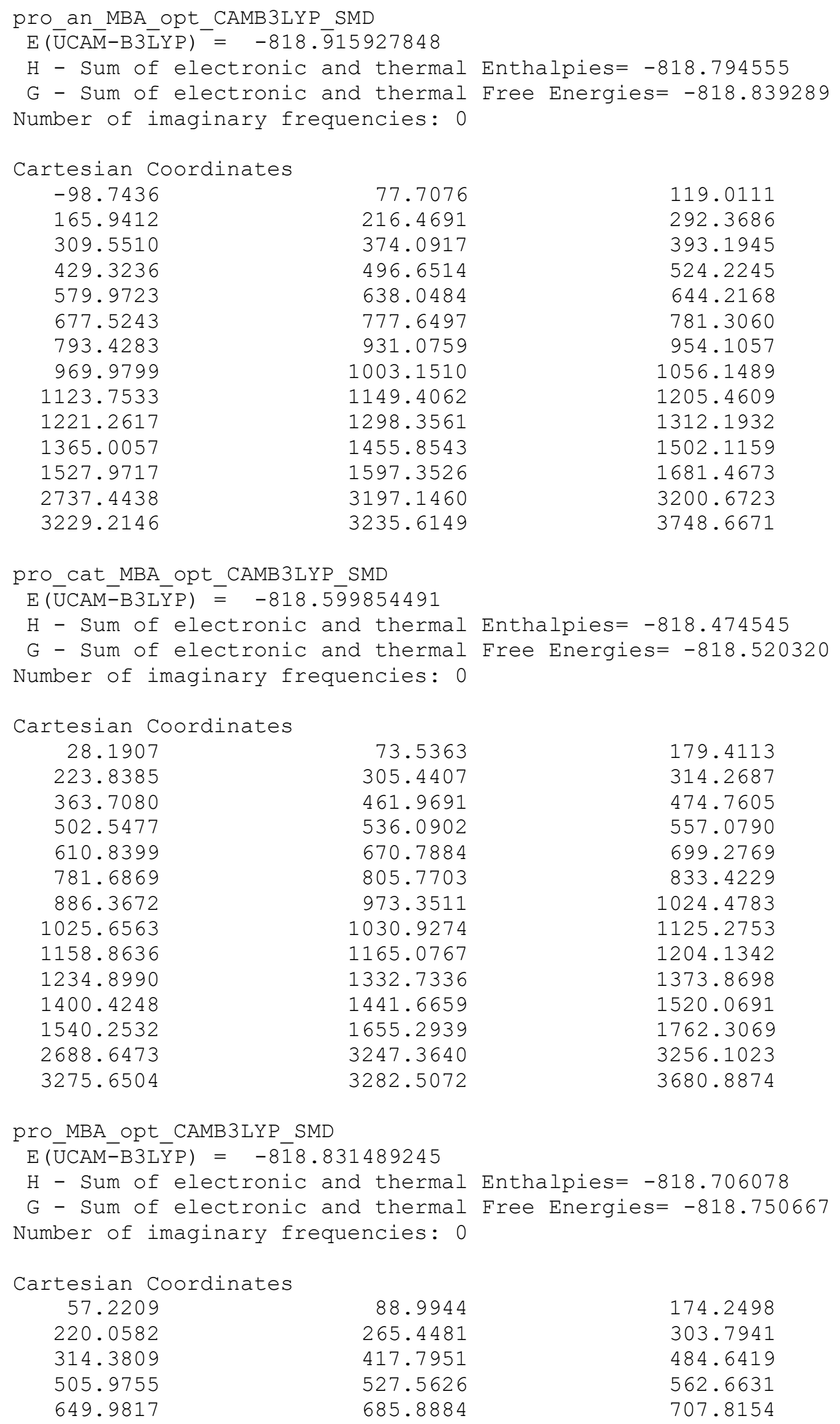


782.4437

875.5176

1018.6857

1138.7583

1236.7242

1386.7707

1645.3848

2736.4036

3252.0989
809.9590

955.5404

1039.8448

1156.0689

1342.4300

1465.3276

1679.2456

3227.8754

3260.4608
861.2074

1008.1082

1114.5290

1199.9358

1354.3487

1556.6211

1730.6418

3233.5920

3699.4882 


\section{References:}

1. Scarpitti, B. T.; Morrison, A. M.; Buyanova, M.; Schultz, Z. D., Comparison of 4-Mercaptobenzoic Acid Surface-Enhanced Raman Spectroscopy-Based Methods for $\mathrm{pH}$ Determination in Cells. Appl Spectrosc 2020, 74 (11), 1423-1432.

2. $\quad$ Ohio Supercomputer Center. 1987. http://osc.edu/ark:/19495/f5s 1ph73

3. Frisch, M. J.; Trucks, G. W.; Schlegel, H. B.; Scuseria, G. E.; Robb, M. A.; Cheeseman, J. R.; Scalmani, G.; Barone, V.; Petersson, G. A.; Nakatsuji, H.; Li, X.; Caricato, M.; Marenich, A. V.; Bloino, J.; Janesko, B. G.; Gomperts, R.; Mennucci, B.; Hratchian, H. P.; Ortiz, J. V.; Izmaylov, A. F.; Sonnenberg, J. L.; Williams; Ding, F.; Lipparini, F.; Egidi, F.; Goings, J.; Peng, B.; Petrone, A.; Henderson, T.; Ranasinghe, D.; Zakrzewski, V. G.; Gao, J.; Rega, N.; Zheng, G.; Liang, W.; Hada, M.; Ehara, M.; Toyota, K.; Fukuda, R.; Hasegawa, J.; Ishida, M.; Nakajima, T.; Honda, Y.; Kitao, O.; Nakai, H.; Vreven, T.; Throssell, K.; Montgomery Jr., J. A.; Peralta, J. E.; Ogliaro, F.; Bearpark, M. J.; Heyd, J. J.; Brothers, E. N.; Kudin, K. N.; Staroverov, V. N.; Keith, T. A.; Kobayashi, R.; Normand, J.; Raghavachari, K.; Rendell, A. P.; Burant, J. C.; Iyengar, S. S.; Tomasi, J.; Cossi, M.; Millam, J. M.; Klene, M.; Adamo, C.; Cammi, R.; Ochterski, J. W.; Martin, R. L.; Morokuma, K.; Farkas, O.; Foresman, J. B.; Fox, D. J. Gaussian 16 Rev. C.01, Wallingford, CT, 2016.

4. Dennington, R.; Keith, T. A.; Millam, J. M. GaussView, Version 6.1, Shawnee Mission, KS, 2016.

5. Yanai, T.; Tew, D. P.; Handy, N. C., A new hybrid exchange-correlation functional using the Coulomb-attenuating method (CAM-B3LYP). Chem Phys Lett 2004, 393 (1), 51-57.

6. Hehre, W. J.; Ditchfield, R.; Pople, J. A., Self-Consistent Molecular Orbital Methods. XII. Further Extensions of Gaussian-Type Basis Sets for Use in Molecular Orbital Studies of Organic Molecules. The Journal of Chemical Physics 1972, 56 (5), 2257-2261.

7. Hariharan, P. C.; Pople, J. A., Accuracy of AH n equilibrium geometries by single determinant molecular orbital theory. Molecular Physics 1974, 27 (1), 209-214.

8. Hariharan, P. C.; Pople, J. A., The influence of polarization functions on molecular orbital hydrogenation energies. Theoretica chimica acta 1973, 28 (3), 213-222. 9. Miertuš, S.; Scrocco, E.; Tomasi, J., Electrostatic interaction of a solute with a continuum. A direct utilizaion of $\mathrm{AB}$ initio molecular potentials for the prevision of solvent effects. Chemical Physics 1981, 55 (1), 117-129.

10. Miertus̃, S.; Tomasi, J., Approximate evaluations of the electrostatic free energy and internal energy changes in solution processes. Chemical Physics 1982, 65 (2), 239245 .

11. Marenich, A. V.; Cramer, C. J.; Truhlar, D. G., Universal Solvation Model Based on Solute Electron Density and on a Continuum Model of the Solvent Defined by the Bulk Dielectric Constant and Atomic Surface Tensions. The Journal of Physical Chemistry B 2009, 113 (18), 6378-6396.

12. de Albuquerque, C. D. L.; Schultz, Z. D., Super-resolution Surface-Enhanced Raman Scattering Imaging of Single Particles in Cells. Anal. Chem. 2020, 92 (13), 93899398. 